\title{
Capecitabine and Oxaliplatin versus 5-Fluorouracil in the Neoadjuvant Chemoradiation for Patients with Locally Advanced Rectal Carcinoma: A Comparative Study
}

\author{
Amal Rayan 1*, Hosam A. Hasan², Asmaa M. Zahran³, Ahmed Soliman', Khalid Rezk ${ }^{5}$ \\ ${ }^{1}$ Clinical Oncology Department, Faculty of Medicine, Assiut University, Assiut, Egypt \\ ${ }^{2}$ Diagnostic Radiology Department, Faculty of Medicine, Assiut University, Assiut, Egypt \\ ${ }^{3}$ Clinical Pathology Department, South Egypt Cancer Institute, Assiut University, Assiut \\ ${ }^{4}$ General Surgery Departments, Faculty of medicine, Assiut University, Assiut, Egypt \\ ${ }^{5}$ Surgical Oncology Department, South Egypt Cancer Institute, Assiut University, Assiut, Egypt \\ Email: *amal3774rayan@gmail.com
}

How to cite this paper: Rayan, A., Hasan, H.A., Zahran, A.M., Soliman, A. and Rezk, K. (2018) Capecitabine and Oxaliplatin versus 5 -Fluorouracil in the Neoadjuvant Chemoradiation for Patients with Locally Advanced Rectal Carcinoma: A Comparative Study. Journal of Cancer Therapy, 9, 767-779.

https://doi.org/10.4236/jct.2018.910063

Received: June 10, 2018

Accepted: October 5, 2018

Published: October 8, 2018

Copyright $(9) 2018$ by authors and Scientific Research Publishing Inc. This work is licensed under the Creative Commons Attribution International License (CC BY 4.0).

http://creativecommons.org/licenses/by/4.0/

\begin{abstract}
Aim of work: This study aimed to evaluate the resectability rate, rate of conservative surgery, toxicity, local control, and disease free survival for oxaliplatin and capecitabine based chemoradiation compared to standard 5-FU based chemoradiation in locally advanced cancer rectum. Patients and methods: 65 patients were eligible; 33 patients received oxaliplatin and capecitabine based chemoradiation (arm I) and 32 patients received 5-FU based chemoradiation (arm II). Results: The overall response rate in arms I and II were $78.7 \%$ and $87.5 \%$ respectively. Conservative surgery was done in $81.81 \%$ and $53.13 \%$ of patients with arms I and II, pathologic complete response (pCR) rate was significantly better in arm I than arm II (30.3\% vs. $21.9 \%, P<0.01)$. 3 -year recurrence rates were $54.5 \%$ and $56.2 \%$ in arms I, II respectively; the median disease free survival (DFS) were 30 months and 15 months in arms I and II respectively. Grade III anemia, grade IV diarrhea and severe proctitis were developed in a significantly large number of patients with arm I; in addition deep venous thrombosis (DVT) was developed in $15.15 \%$ of patients with arm I but none in arm II. Conclusion: The addition of oxaliplatin to the preoperative chemo radiation increased the response rate mainly pCR rate which was considered a target goal in the neoadjuvant treatment, but it was not recommended because of higher toxicity and no significant effect on DFS in different response groups of arm I when compared to arm II, but longer follow up may be needed to evaluate the overall survival.
\end{abstract}




\section{Keywords}

Neoadjuvant Concurrent Chemoradiation, Oxaliplatin, Capecitabine, Locally Advanced Cancer Rectum

\section{Introduction}

Globally, colorectal cancer is the third most common form of cancer after lung and breast cancers. In 2014, the estimated new cases and deaths from rectal carcinoma in the United States [1] were 40,000 (rectal cancer only) and 50,310 (colon and rectal cancers combined) respectively.

In Egypt, reports showed that colorectal cancer was detected in $11 \%-15 \%$ of patients who underwent colonoscopy and diagnosed in $29 \%-31 \%$ of patients aged $\leq 40$ years [2].

Preoperative concurrent chemo radiation is routinely performed for locally advanced rectal cancer; (T3-T4 and/or N+) to achieve down staging of the tumor and soto increase its resectability rate. Other benefits of preoperative chemo radiation are to increase the probability of a sphincter-saving procedure, reduce the risk of local recurrence, and thereby, improve the survival [3].

A number of well randomized controlled trials had been made to evaluate the impact of oxaliplatin when added to 5-FU or capecitabine based chemo radiation on different response rates especially $\mathrm{pCR}$ rate and on the toxicity pattern in patients with cT3-4 and/or N+ rectal adenocarcinoma. In the STAR-01 trial 747 patients were randomized to preoperative chemo radiation with $50.4 \mathrm{~Gy}+\mathrm{CI}$ 5-FU with or without weekly oxaliplatin [4]. Grade 3 toxicity with oxaliplatin was significantly higher $(24 \%$ vs. $8 \%, P<0.001)$ with no improvement in the pCR rate ( $15 \%$ vs. $16 \%)$.

In the ACCORD 12/0405 PRODIGIE trial, patients were randomized to preoperative chemo radiation with radiotherapy dose of 50 Gy concurrently with capecitabine and oxaliplatin (CAPOX) andradiotherapy dose of 45 Gy concurrently with capecitabine [5]. Similar findings like STAR-01 trial was achieved; in addition, oxaliplatin did not improve the 3-year local recurrence (6\% vs. $4 \%$ ) or the overall survival ( $88 \%$ vs. $83 \%$ ) [6].

The NSABP R-0 4 trial which was a 4 arms trial $(2 \times 2$ comparison $)$ of continuous infusion 5-FU based chemo radiation vs. capecitabine based preoperative chemo radiation (50.4 Gy) with or without oxaliplatin [7] concluded that the addition of oxaliplatin (to either 5-FU or capecitabine) was associated with significantly higher incidence of grade 3 diarrhea ( $15 \%$ vs. $7 \%, P=0.0001)$ and no improvement in the incidence of pCR (21\% vs. $19 \%)$ or sphincter-sparing surgery ( $60 \%$ vs. $64 \%)$.

However; the German CAO/ARO/AIO-04 [8] proved that oxaliplatin based chemo-radiation produced a significant improvement in pCR (17\% vs. $13 \%, P=$ 0.045 ) with no corresponding increase in acute grade $\geq 3$ toxicity ( $23 \%$ vs. $22 \%$ ).

As 3 of 4 randomized trials revealed an increase in the acute toxicity with no 
real benefit in the pCR rate so the current preoperative chemoradiation regimensdon't include oxaliplatin.

We aimed to evaluate the resectability rate, rate of conservative surgery, toxicity profile, local control, and disease-free survival for oxaliplatin and capecitabine-based chemoradiation in comparison to standard 5 FU-based chemoradiation in locally advanced cancer rectum.

\section{Patients and Methods}

This study was a randomized controlled two arm trialand was conducted from June 2012 to September 2013 at Clinical Oncology, Surgical, and Diagnostic Radiology departments of Assiut University Hospital and clinical pathology and surgical oncology departments of south Egypt cancer institute, to compare capecitabine and oxaliplatin based chemoradiation to 5-fluorouracil based chemoradiation as regard treatment outcomes and toxicity patterns for patients with locally advanced cancer rectum.

All patients met the following eligibility criteria:

- Age > 18 years;

- Eastern Cooperative Oncology Group performance status (ECOG PS) $\leq 2$;

- Histologically proven rectal adenocarcinoma TNM staging: T3-T4, Nx M0, any $\mathrm{T}$ any $\mathrm{N}+\mathrm{M} 0$ disease;

- Adequate bone marrow functions with total leucocytic count $>3000 / \mathrm{mm}^{3}$, platelet count $>100,000 / \mathrm{mm}^{3}$, hemoglobin level $>11 \mathrm{gm} / \mathrm{dL}$, normal hepatic and renal functions (LFTs, RFTs);

- Patients with temporary colostomy were allowed;

- No previous chemotherapy or pelvic irradiation;

- Pregnant or lactating females weren't included;

- Written consent from all eligible patients was taken. All eligible patients underwent the followings:

- History, physical examination, digital rectal examination;

- Complete blood picture (CBC), blood chemistries (RFTs, LFTs, electrolytes), carcinoembryonic antigen (CEA);

- Pelvic magnetic resonance imaging (MRI), multisclice computed tomography (MSCT) chest and abdomen;

- Colonoscopy to exclude other primaries;

- Proctoscopy and biopsy.

Protocol of concurrent chemo-radiation:

Patients were randomly assigned either to receive arm I of capecitabine and oxaliplatin based chemoradiation or arm II of standard 5 FU-based chemoradiation.

\subsection{Chemotherapy}

Arm I: oxaliplatin $45 \mathrm{mg} / \mathrm{m}^{2}$ was given weekly over $2 \mathrm{~h}$ infusion and $2 \mathrm{~h}$ just before radiation setting. capecitabine $825 \mathrm{mg} / \mathrm{m}^{2} /$ dose given twice daily (total $=$ $1650 \mathrm{mg} / \mathrm{m}^{2}$ daily), the 1 st daily dose was given $2 \mathrm{~h}$ before radiation setting, five 
days per week, and chemotherapy continued throughout the radiotherapy course.

Arm II: 5-FU $225 \mathrm{mg} / \mathrm{m}^{2}$ was given over 24 hours infusion for 5 days/week during radiotherapy $(\mathrm{RT})$.

\subsection{Radiotherapy Protocol}

The treatment was given by $3 \mathrm{D}$ conformal RT for both arms in two phases. Clinical target volume (CTV) for phase I included the tumor, presacral lymph nodes (LN), perirectal LN, external and internal iliac LNs, and inguinal LN for tumors extending below the dentate line, while CTV for phase II included the tumor $+3 \mathrm{~cm}$ safety margin. The dose for phase I; $45 \mathrm{~Gy} / 25$ fractions/5 weeks, and for phase II; $5.4 \mathrm{~Gy} / 3$ fractions/half a week. We limited the dose to the small bowel to 45 - $50 \mathrm{~Gy}$, and the femoral head and neck to $42 \mathrm{~Gy}$.

\subsection{Evaluation of Treatment Response}

During the treatment: repeated $\mathrm{CBC}$ and renal function were done, follow up of improvement of symptoms and monitoring of development of toxicity occurred during progress of therapy were done, and the toxicities were recorded according to National Cancer Institute Common Terminology Criteria for Adverse Events version 3 (NCI CTCAEs). Assessment of the response was done within 6 weeks after therapy mainly by imaging; high resolution pelvic MRI 1.5 tesla, MSCT chest and abdomen with contrast using 64 row slices scanner from the root of the neck to pelvic floor. Assessment was done based on response evaluation criteria in solid tumors (RECIST) 1 criteria with scan thickness of $\leq 5 \mathrm{~mm}$,

Evaluation of target lesions:

Complete Response (CR): disappearance of all target lesions, any pathological LN must have a short axis of $<10 \mathrm{~mm}$ in diameter whether target or nontarget LNs.

Partial Response (PR): at least 30\% reduction in the sum of diameters of target lesions, in comparison to baseline sum diameters.

Progressive Disease (PD): at least 20\% increase in the sum of diameters of target lesions; any new lesion is considered PD.

Stable Disease (SD): neither PD nor PR based on the reference of the smallest sum diameters.

Residual Disease was defined based on depth of invasion, distance from anal verge, number of enlarged LNs, site of the primary tumor, mesorectal infiltration. Trans-rectal U/S was done in some cases to evaluate the depth of invasion before surgery. Evaluation of the response also was done through repeated measurement of CEA, CBC, blood chemistries.

Surgery was done 6 weeks later after the end of chemo-radiation and based on the results of clinical, imaging, and laboratory evaluations of the response, in some cases temporary colostomy was done to relieve the obstruction before chemo-radiation, sphinecteric saving surgeries included trans-anal excision, low ante- 
rior resection, and coloanal anastomosis, and radical abdominoperineal resection.

\subsection{Statistical Analysis}

The response was evaluated as mentioned based on RECIST 1 criteria, Disease free survival (DFS) was determined from the time of diagnosis to the time of locoregional relapse (the use of DFS rather than overall survival (OS) has become more frequent in adjuvant cancer trials) [9]. DFS offers earlier presentation of data as disease recurrence occurs earlier than death from the disease. Besides; there are more events in DFS than in OS, like locoregional recurrence. Local recurrence (LR) was determined clinically or radiologically but proven by biopsy. Chi-square test was used to compare differences in distribution of frequencies among various groups of response, Mann-Whitney test was used to compare different quantitative data, Anova test was used to determine the significance between the means of different groups. $P$-value of 0.05 was considered significant at $95 \%$ confidence interval. DFS was calculated using Kaplan-Meier [10], Log rank test was used to test the effect of different risk factors on survival. All data were computerized and analyzed with SPSS ver. 21.

\section{Results}

The present study was done at Assiut University Hospital and South Egypt Cancer Institute, and was approved by the ethical committee of institutional review board of Assiut faculty of medicine. 65 patients were accrued from June 2012 to September 2013, 33 patients were included in arm I and 32 patients in arm II.

\subsection{Demographic Data of Patients}

The mean age for arm I and II were $42.48,40.16$ respectively, male to female ratio were 1.2:1, 0.68:1 for arms I and II respectively. $30 \%$ of patients were of ECOG PS 1 in arm I but 53. 1\% of patients were in PS 2 in arm II (Table 1).

\subsection{Clinical Presentation}

The main presenting symptom was bleeding per rectum and encountered in $66.7 \%$, and $68.8 \%$ of patients in arm I and II respectively (22 patients in each arm), abdominal pain was reported in $51.5 \%$ of patients in arm I (17 patients) and $18.7 \%$ (6 patients) of arm II, altered bowel habits in $45 \%$ of patients (15 patients) in arm I and $9.4 \%$ of patients of arm II (3 patients), and intestinal obstruction was presented in $9 \%$ and $9.4 \%$ of patients with arm I and II respectively (3 patients in each arm).

\subsection{Pathological and Radiological Characteristics of Patients}

The most common adenocarcinoma subtype in arms I and II were conventional type reported in 70\% (23 patients) and $81.25 \%$ (26 patients) respectively. Tumors arose from mid-rectum were most common in arm I while tumors arose from lower rectum were most common in arm II (Table 1). 


\subsection{Carcinoembryonic Antigen Level at Presentation}

At presentation; CEA was elevated in $63.63 \%$ (21 patients), $34.4 \%$ (11 patients) of arms I and II respectively.

\subsection{Type of Surgery}

Conservative surgery was done in $81.81 \%$ (27 patients) of patients with arm I, while it was done in $53.13 \%$ (17 patients) of patients with arm II, and the results were significant for arm I $(P<0.001)$ (Table 2).

\subsection{Response to Treatment}

The most common pattern of response based on post chemoradiation MRI; in arm I was complete response and developed in 54.5\% (18 patients), but it was partial response in $50 \%$ (16 patients) of arm II. The overall response rate in arms I and II were $78.7 \%$ and $87.5 \%$ respectively and the response was significantly better for arm I $(P<0.01)$ (Table 3$)$.

The rates of pathological complete response after surgery as defined by Ryan staging of pathological response were $30.3 \%$ and $21.9 \%$ in arms I and II respectively which was significantly better in arm I than arm II $(P<0.01)$.

\subsection{Distribution of Conservative Surgery over Different Response Groups}

$51.85 \%$ of patients (14 patients) with arm I who developed CR were underwent SP, while $52.9 \%$ (9 patients) of arm II with CR were underwent SP (the percentage was higher in arm II but the actual number was higher in arm I) the results were also significantly better for arm I than arm II $(P<0.01)$ (Table 4).

\subsection{Local Control among Study Groups}

3-year local control rate was $45.5 \%$ in arm I (15/33 patients) and it was slightly higher than arm II which was $43.8 \%$ (14/32 patients).

\subsection{Disease Free Survival (DFS)}

Over a period of follow up of more than 40 months for the entire cohort, local recurrence was detected in 18 patients in each arm, so the 3-year recurrence rates were $54.5 \%$ and $56.2 \%$ in arms I, II respectively.

The mean DFS \pm SE were $26.28 \pm 1.93$ and $17.03 \pm 1.98$ in arms I and II respectively, while the median DFS in arm I was 30 months (Figure 1) which was higher than that of arm II as it was 15 months (Figure 2).

\subsection{Relation between Response and DFS}

There was a significant relation between the response and DFS in arms I and II $(P<0.01$ arm I and $P<0.000$ in arm II) but no significant difference between DFS of both arms in any response group (Table 5). 


\subsection{Toxicity among Different Study Groups}

Toxicities among patients treated with arm I were significantly higher than toxicities among patients treated with arm II as severe proctitis developed in 9.1\% of patients ( 3 patients), grade III anemia developed in $6.1 \%$ of patients ( 2 patients), grade IV diarrhea was developed in one patient (in arm I). Deep venous thrombosis (DVT) was developed in $15.15 \%$ of patients with arm I (5 patients) but none developed DVT in arm II (Table 6).

Table 1. Characteristics of patients.

\begin{tabular}{|c|c|c|c|c|}
\hline Data & Arm I & & Arm II & \\
\hline & $\mathrm{N}$ & $\%$ & $\mathrm{~N}$ & $\%$ \\
\hline Age $($ mean $\pm S D)$ & $42.48 \pm 16.3$ & & $40.16 \pm 10.33$ & \\
\hline Min - max & $(17-80)$ & & $(20-60)$ & \\
\hline \multicolumn{5}{|l|}{$\underline{\text { Sex }}$} \\
\hline Male & 18 & $54.5 \%$ & 13 & $40.6 \%$ \\
\hline Female & 15 & $45.5 \%$ & 19 & $59.4 \%$ \\
\hline \multicolumn{5}{|l|}{ ECOG PS } \\
\hline 0 & 9 & $27.3 \%$ & 2 & $6.25 \%$ \\
\hline 1 & 10 & $30.3 \%$ & 11 & $34.4 \%$ \\
\hline 2 & 8 & $24.2 \%$ & 17 & $53.1 \%$ \\
\hline 3 & 6 & $18.2 \%$ & 2 & $6.25 \%$ \\
\hline \multicolumn{5}{|l|}{ Adenocarcinoma } \\
\hline -Conventional type & 23 & $70 \%$ & 26 & $81.25 \%$ \\
\hline -Mucinous type & 5 & $15 \%$ & 4 & $12.4 \%$ \\
\hline -Signet ring type & 5 & $15 \%$ & 2 & $6.2 \%$ \\
\hline \multicolumn{5}{|l|}{$\underline{\text { Grading }}$} \\
\hline G1 & 15 & $45.4 \%$ & 7 & $21.9 \%$ \\
\hline G2 & 9 & $27.3 \%$ & 14 & $43.8 \%$ \\
\hline G3 & 9 & $27.3 \%$ & 3 & $9.4 \%$ \\
\hline G4 & 0 & $0 \%$ & 8 & $25 \%$ \\
\hline \multicolumn{5}{|l|}{ T stage } \\
\hline $\mathrm{T} 2$ & 10 & $30.3 \%$ & 2 & $6.2 \%$ \\
\hline $\mathrm{T} 3$ & 14 & $42.42 \%$ & 15 & $46.9 \%$ \\
\hline $\mathrm{T} 4$ & 9 & $27.27 \%$ & 15 & $46.9 \%$ \\
\hline \multicolumn{5}{|l|}{ N stage } \\
\hline No & 15 & $45.45 \%$ & 15 & $46.9 \%$ \\
\hline $\mathrm{N}+$ & 18 & $54.54 \%$ & 17 & $53.1 \%$ \\
\hline \multicolumn{5}{|c|}{ Tumor localization from anal verge } \\
\hline Lower rectum & 12 & $36.36 \%$ & 23 & $71.9 \%$ \\
\hline Mid-rectum & 16 & $48.48 \%$ & 4 & $12.5 \%$ \\
\hline Upper rectum & 5 & $15.15 \%$ & 5 & $15.6 \%$ \\
\hline
\end{tabular}

$\mathrm{N}=$ node, $\mathrm{T}=$ tumor, $\mathrm{G}=$ grade. 
Table 2. The type of surgery.

\begin{tabular}{llllll}
\hline Surgery & $\begin{array}{l}\text { Arm I } \\
\text { No }\end{array}$ & $\begin{array}{c}\text { total }=33 \\
\%\end{array}$ & $\begin{array}{l}\text { Arm II } \\
\text { No }\end{array}$ & $\begin{array}{c}\text { total }=32 \\
\%\end{array}$ & $P$ value \\
\hline Conservative & 27 & $81.81 \%$ & 17 & $53.13 \%$ & $P<0.001^{* * *}$ \\
$($ APR $)$ & 6 & $18.18 \%$ & 15 & $46.87 \%$ & \\
\hline
\end{tabular}

$\mathrm{APR}=$ abdominoperineal resection, ${ }^{* * *}=$ highly significant.

Table 3. Response to treatment.

\begin{tabular}{llllll}
\hline Response & $\begin{array}{l}\text { Arm I } \\
\text { No }\end{array}$ & $\%$ & $\begin{array}{l}\text { Arm II } \\
\text { No }\end{array}$ & $\%$ & P Value \\
\hline -CR & 18 & $54.5 \%$ & 12 & $37.5 \%$ & \\
-PR & 8 & $24.2 \%$ & 16 & $50 \%$ & $P<0.01^{\star}$ \\
-SD & 7 & $21.3 \%$ & 4 & $12.5 \%$ & \\
-Pathologic CR & 10 & $30.3 \%$ & 7 & $21.9 \%$ & \\
\hline
\end{tabular}

* = significant, $\mathrm{CR}=$ complete response, $\mathrm{PR}=$ partial response, $\mathrm{SD}=$ stable disease.

Table 4. Rate of SP among different response groups.

\begin{tabular}{llllll}
\hline \multirow{2}{*}{ Response } & \multicolumn{5}{l}{ Rate of sphincter preservation (SP) } \\
\cline { 2 - 6 } & No & Arm I & No & Arm II & Pvalue \\
\hline CR & 14 & $51.85 \%$ & 9 & $52.9 \%$ & \\
PR & 6 & $22.22 \%$ & 7 & $41.2 \%$ & $P<0.01^{*}$ \\
SD & 7 & $25.93 \%$ & 1 & $5.9 \%$ & \\
\hline
\end{tabular}

* = significant, $\mathrm{CR}=$ complete response, $\mathrm{PR}=$ partial response, $\mathrm{SD}=$ stable disease.

Table 5. Relation between the response and DFS.

\begin{tabular}{llll}
\hline $\begin{array}{lll}\text { Item } \\
\text { response }\end{array}$ & DFS & DFS & $P$ value \\
\hline CR & Arm I & Arm II & \\
PR & $29.16 \pm 2.52$ & $24.41 \pm 9.37$ & $P=0.376$ n.s \\
SD & $23.04 \pm 3.84$ & $22.75 \pm 8.62$ & \\
$P$ value & $16.32 \pm 3.06$ & 0.0 & $P=0.382$ n.s \\
\hline
\end{tabular}

DFS $=$ disease-free survival, n.s $=$ nonsignificant, ${ }^{*}=$ significant, ${ }^{* * *}=$ highly significant, $\mathrm{CR}=$ complete response, $\mathrm{PR}=$ partial response, $\mathrm{SD}=$ stable disease.

Table 6. Toxicity among patients treated with pre-operative chemoradiation.

\begin{tabular}{|c|c|c|c|c|c|}
\hline Toxicity & Arm I & & Arm II & & $P$ value \\
\hline \multicolumn{6}{|c|}{ Acute proctitis } \\
\hline -Mild & 18 & $54.54 \%$ & $721.9 \%$ & & \\
\hline -Moderate & 12 & $36.36 \%$ & $825.0 \%$ & & $<0.000^{* * *}$ \\
\hline -Severe & 3 & $9.1 \%$ & 0 & $0 \%$ & \\
\hline \multicolumn{6}{|l|}{-Anemia } \\
\hline$-\mathrm{G} 1$ & 6 & $18.18 \%$ & $1031.25 \%$ & & \\
\hline$-\mathrm{G} 2$ & 9 & $27.27 \%$ & $13.1 \%$ & & $<0.001^{\star * *}$ \\
\hline$-G 3$ & 2 & $6.1 \%$ & 0 & $0 \%$ & \\
\hline
\end{tabular}




\section{Continued}

\section{- Diarrhea}

\begin{tabular}{|c|c|c|c|c|c|}
\hline$-\mathrm{G} 1$ & 10 & $30.3 \%$ & $515.62 \%$ & & \\
\hline$-G 2$ & 7 & $21.21 \%$ & $515.62 \%$ & & $<0.02^{*}$ \\
\hline$-G 3$ & 1 & $3.03 \%$ & 1 & $3.0 \%$ & \\
\hline$-\mathrm{G} 4$ & 1 & $3.03 \%$ & 0 & $0 \%$ & \\
\hline - Neutropenia & 5 & $15.15 \%$ & $13.1 \%$ & & $<0.04^{\star}$ \\
\hline -Hand \& Foot syndrome & 7 & $21.21 \%$ & $26.2 \%$ & & $<0.04^{\star}$ \\
\hline$\bullet$ DVT & 5 & $15.15 \%$ & 0 & $0 \%$ & $0.271 \mathrm{n} . \mathrm{s}$ \\
\hline
\end{tabular}

$\mathrm{G}=$ grade, $\mathrm{n} . \mathrm{s}=$ non significant, ${ }^{*}=$ significant, ${ }^{* * *}=$ highly significant, DVT $=$ deep venous thrombosis.

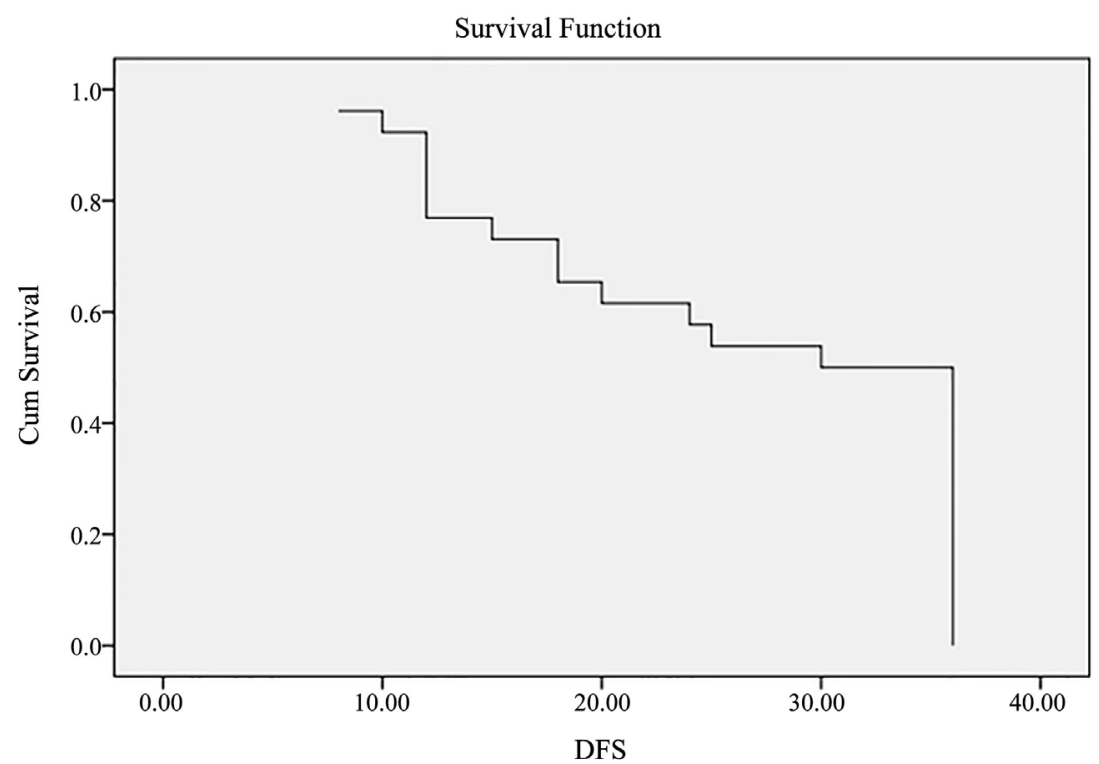

Figure 1. DFS for 33 patients treated with capecitabine and oxaliplatin based chemoradiation (arm I). In month.

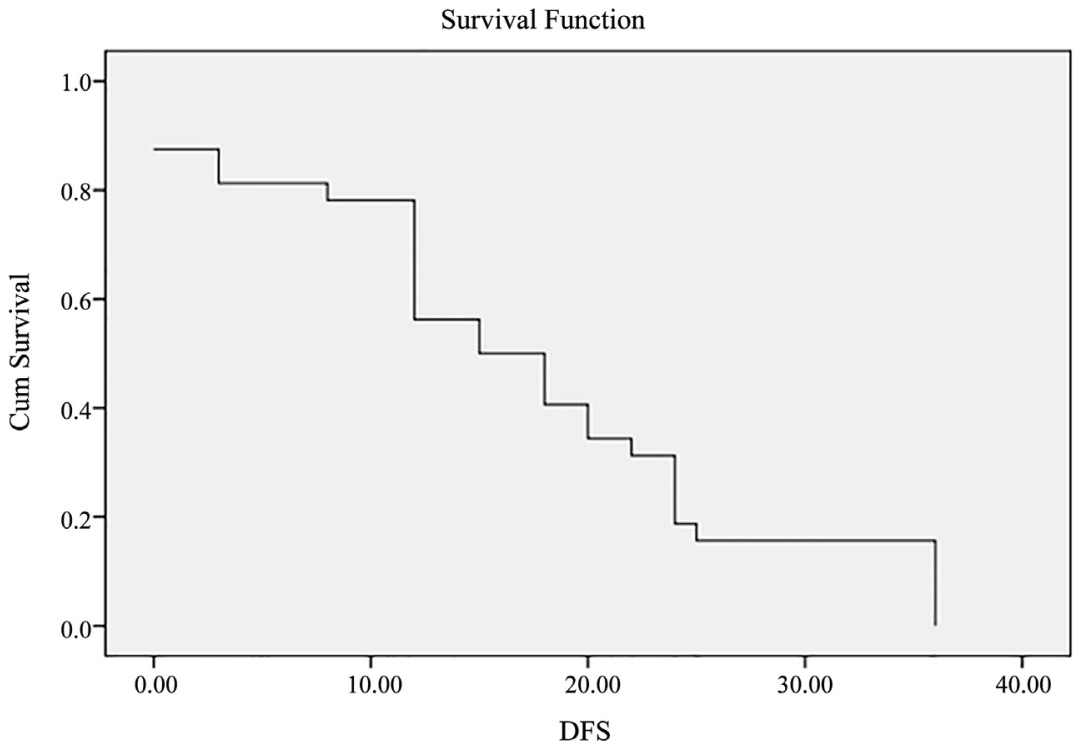

Figure 2. DFS in 32 patients treated with continuous 5-FU based concurrent chemoradiation (arm II). In month. 


\section{Discussion}

5 -flourouracil is currently the standard cytotoxic agent for preoperative chemo radiotherapy, however; considering the disadvantages of continuous infusion, a number of studies evaluatedother cytotoxic agents. These are mainly oral agents like capecitabine, and these studies concludedthat no significant difference in treatment outcomes and more favorable toxicity when compared to CI 5-FU. Preoperative chemoradiation with concurrent capecitabine is considered astandard treatment for locally advanced rectal adenocarcinoma [11] to avoid local relapse and improve survival.

Oxaliplatin is used for preoperative concurrent chemo radiation because of its radiosensitizing properties detected in preclinical studies [12]; and numerous phase II studies combining this agent with fluoropyrimidine-based chemoradiation reported pathologic complete response rates ranging from $14 \%$ to $30 \%$. Many studies demonstrated a positive correlation between pathologic complete response rate and different endpoints as distant metastasis-free survival (DMFS), DFS, and OS [13].

In the German CAO/ARO/AIO-04 trial [8]; the results demonstrated a significantly higher pathologic complete response $(17 \%$ vs. $13 \%, P=0.038)$ with the addition of oxaliplatin to 5-FU based chemoradiation with no significant differences in grades 3 or 4 between arms.

In our study, the results were comparable to the German trial, the percentage of patients achieved pCR was significantly higher in arm I than arm II to an extent sufficiently translated into significantly higher percentage of patients underwent conservative surgery ( $81.18 \%$ vs. $53.13 \% P<0.01)$, but in contrast to the German trial; grade 3 and 4 toxicities were significantly higher in those receiving oxaliplatin.

The response to neoadjuvant chemo radiotherapy is a strong predictor of DFS [14] and this was evident in a subgroup of patients from the German rectal cancer trial, 5-year DFS ranged from $86 \%$ for complete response to $75 \%$ for patients with tumor response of $25 \%-75 \%$, and $63 \%$ for patients with $<25 \%$ tumor response $(P=0.006)[15]$.

This relation between the response and DFS was also evident in our trial as patients achieved CR was associated with significantly better DFS in both arms $(P<0.01, P<0.000$ in arms I and II respectively), but no significant difference between DFS in different response groups of both arms and no significant differences in DFS of both arms which wasn't comparable to updated results of the German CAO/ARO/AIO-04 randomized phase III trial which concluded that the addition of oxaliplatin to 5-FU based neoadjuvant chemoradiation with adjuvant chemotherapy in locally advanced cancer rectum significantly improved 3-year DFS $(P=0.03)[16]$.

In RadiOxCape trial; Machiels et al concluded that the concurrent chemoradiation with RT dose $50 \mathrm{~Gy}$ and capecitabine plus oxaliplatin was associated with $30 \%$ incidence of diarrhea, $14 \%$ for pCR rate and better down staging [17], and 
our results were not comparable to these results as diarrhea with all grades developed in $57.6 \%$ of patients with arm I and $34.24 \%$ of patients with arm II. And pCR rate were $30.3 \%$ and $21.9 \%$ in arms I and II respectively.

Borschitz et al. [18] reported local recurrence rates by pathologic stage: ypT1: 2\%; ypT2: $6 \%$ - 20\%. The incidence was $43 \%$ in ypT3 tumors which did not respond to preoperative chemoradiation. In our patients; local recurrence rate was very high in both arms it is $54.5 \%$ and $56.2 \%$ in arms I and II respectively, possibly because of higher percentage of T3-4 lesions (69.7\% and 71.9\% in arms I, II), large percentage of patients did not respond to treatment (SD in $21.3 \%$ and $12.5 \%$ of arms I and II respectively), and $36.4 \%$ and $71.9 \%$ of patients in arms I, II had distal rectal tumors with a possibility of inadequate resection margins.

The side effects of preoperative chemoradiation for cancer rectum may affect patients' quality of life and in our study; oxaliplatin and capecitabine based chemoradiation was associated with significantly higher toxicity that was comparable to different literatures and previous studies. DVT occurred in $15.15 \%$ of patients in oxaliplatin group and this toxicity is a rare one and not mentioned in many trials, but it may be attributed to long postoperative recovery period in some patients, previous risk factors like diabetes, obesity, previous DVT, chronic liver disease.

There are a number of weak points in our study including the small number of patients to which attributed the difference between our results and international studies, and our patients came from only two institutions so the results were not generalized.

\section{Conclusion}

The addition of oxaliplatin to the preoperative chemoradiation increases the response rate especially $\mathrm{pCR}$ rate which was considered a target goal in the neoadjuvant treatment, but it was not recommended because of higher toxicity, and it added no significant effect on DFS in different response groups of both arms when compared to CI 5-FU based chemoradiation, but longer follow up may be needed to evaluate the overall survival.

\section{Conflicts of Interest}

The authors declare no conflicts of interest regarding the publication of this paper.

\section{References}

[1] American Cancer Society (2014) Cancer Facts \& Figures. Atlanta.

[2] Elbatea, H., Enaba, M., Elkassas, G., et al. (2011) Indication and Outcome of Colonoscopy in the Middle of Nile Delta of Egypt. Digestive Diseases and Sciences, 56, 2120-2123. https://doi.org/10.1007/s10620-010-1538-1

[3] Garcia-Aguilar, J., Hernandez de Anda, E., Sirivongs, P., et al. (2003) A Pathologic Complete Response to Preoperative Chemo Radiation Is Associated with Lower Local Recurrence and Improved Survival in Rectal Cancer Patients Treated by 
Mesorectal Excision. Diseases of the Colon \& Rectum, 46, 298-304. https://doi.org/10.1007/s10350-004-6545-x

[4] Aschele, C., Cionini, L., Lonardi, S., et al. (2011) Primary Tumor Response to Preoperative Chemoradiation with or without Oxaliplatin in Locally Advanced Rectal Cancer: Pathologic Results of the STAR-01 Randomized Phase III Trial. Journal of Clinical Oncology, 29, 2773-2780. https://doi.org/10.1200/JCO.2010.34.4911

[5] Gérard, J.P., Azria, D., Gourgou-Bourgade, S., et al. (2010) Comparison of Two Neoadjuvant Chemoradiotherapy Regimens for Locally Advanced Rectal Cancer: Results of the Phase III Trial ACCORD 12/0405-Prodige 2. Journal of Clinical Oncology, 28, 1638-1644. https://doi.org/10.1200/JCO.2009.25.8376

[6] Gérard, J.P., Azria, D., Gourgou-Bourgade, S., et al. (2012) Clinical Outcome of the ACCORD 12/0405 PRODIGE 2 Randomized Trial in Rectal Cancer. Journal of Clinical Oncology, 30, 4558-4565. https://doi.org/10.1200/JCO.2012.42.8771

[7] Fernandez-Martos, C., Nogue, M., Cejas, P., Moreno-Garcia, V., Machancoses, A.H. and Feliu, J. (2012) The Role of Capecitabine in Locally Advanced Rectal Cancer Treatment: An Update. Drugs, 72, 1057-1073. https://doi.org/10.2165/11633870-000000000-00000

[8] Rödel, C., Liersch, T., Becker, H., et al. (2012) Preoperative Chemoradiotherapy and Postoperative Chemotherapy with Fluorouracil and Oxaliplatin versus Fluorouracil Alone in Locally Advanced Rectal Cancer: Initial Results of the German CAO/ARO/AIO-04 Randomized Phase 3 Trial. The Lancet Oncology, 13, 679-687. https://doi.org/10.1016/S1470-2045(12)70187-0

[9] Sargent, D.J., Wieand, H.S., Haller, D.G., et al. (2005) Disease-Free Survival versus Overall Survival as a Primaryend Point for Adjuvant Colon Cancer Studies: Individual Patient Data from 20,898 Patients on 18 Randomized Trials. Journal of Clinical Oncology, 23, 8664-8670. https://doi.org/10.1200/JCO.2005.01.6071

[10] Kaplan, E.L. and Meier, P. (1958) Non-Parametric Estimation for Incomplete Observation. Journal of the American Statistical Association, 53, 457-481. https://doi.org/10.1080/01621459.1958.10501452

[11] Ausili Cefaro, G., Genovesi, D., Vinciguerra, A., et al. (2012) Effects of Preoperative Radiochemotherapy with Capecitabine for Resectable Locally Advanced Rectal Cancer in Elderly Patients. Tumori Journal, 98, 622-629. https://doi.org/10.1177/030089161209800513

[12] Cividalli, A., Ceciarelli, F., Livdi, E., et al. (2002) Radiosensitization by Oxaliplatin in a Mouse Adenocarcinoma: Influence of Treatment Schedule. International Journal of Radiation Oncology, Biology, Physics, 52, 1092-1098. https://doi.org/10.1016/S0360-3016(01)02792-4

[13] Valentini, V., Coco, C., Minsky, B.D., et al. (2008) Randomized, Multicenter, Phase IIb Study of Preoperative Chemoradiotherapy in T3 Mid-Distal Rectal Cancer: Raltitrexed + Oxaliplatin + Radiotherapy versus Cisplatin + 5-Fluorouracil + Radiotherapy. International Journal of Radiation Oncology, Biology, Physics, 70, 403-412.

[14] Quah, H.M., Chou, J.F., Gonen, M., et al. (2008) Pathologic Stage Is Most Prognostic of Disease-Free Survival in Locally Advanced Rectal Cancer Patients after Preoperative Chemoradiation. Cancer, 113, 57-64. https://doi.org/10.1002/cncr.23516

[15] Liersch, T., Langer, C., Ghadimi, B.M., et al. (2006) Lymph Node Status and TS Gene Expression Are Prognostic Markers in Stage II/III Rectal Cancer after Neoadjuvant Fluorouracil-Based Chemoradiotherapy. Journal of Clinical Oncology, 24, 4062-4068. https://doi.org/10.1200/JCO.2005.04.2739

[16] Rodel, C., Liersch, T., Fietkau, R., et al. (2004) Preoperative Chemoradiotherapy 
and Postoperative Chemotherapy with 5-Fluorouracil and Oxaliplatin versus 5-Fluorouracil Alone in Locally Advanced Cancer Rectum: Results of the German CAO/ARO/AIO-04 Randomized Phase III Trial. Journal of Clinical Oncology, 32, 3500-3500.

[17] Machiels, J.P., Duck, L., Honhon, B., et al. (2005) Phase II Study of Preoperative Oxaliplatin, Capecitabine and External Beam Radiotherapy in Patients with Rectal Cancer: The RadiOxCape Study. Annals of Oncology, 16, 1898-1905.

https://doi.org/10.1093/annonc/mdi406

[18] Borschitz, T., Wachtlin, D., Möhler, M., et al. (2008) Neoadjuvant Chemoradiation and Local Excision for T2-3 Rectal Cancer. Annals of Surgical Oncology, 15, 712-720. https://doi.org/10.1245/s10434-007-9732-x 